\title{
Specific biochemical amniotic fluid pattern of fetal isolated esophageal atresia
}

\author{
Cécile Muller', Isabelle Czerkiewicz ${ }^{2}$, Fabien Guimiot ${ }^{3}$, Sophie Dreux², Laurent J. Salomon ${ }^{4}$, Naziha Khen-Dunlop ${ }^{5}$, \\ Arnaud Bonnard ${ }^{1}$, Thomas Schmitz ${ }^{6}$, Jean-François Oury ${ }^{6}$ and Françoise Muller ${ }^{2}$
}

BACKGROUND: Perinatal care of esophageal atresia (EA) may be improved by prenatal diagnosis. Ultrasound findings (polyhydramnios and/or nonvisualization of fetal stomach) lead to a detection rate of $\sim 50 \%$. An amniotic fluid (AF) biochemical pattern characterized by high total protein, $\gamma$-glutamyl transpeptidase (GGTP), and normal L-leucine-aminopeptidase (AMP) leads to a $100 \%$ detection rate. The aim of this study was to explain this specific pattern.

METHODS: On the basis of enzyme activities assay, the following four objectives were sought: (i) comparing AF markers between EA and other digestive tract atresias, (ii) determining local GGTP synthesis in the esophagus (immunohistobiochemistry), (iii) determining the presence of a specific AF-AMP activity inhibitor, and (iv) comparing AF-AMP and AF-GGTP half-lives.

RESULTS: The AF-EA pattern was similar to that observed in upper duodenal atresia (above the Oddi sphincter). No local synthesis of GGTP was observed in the esophagus. No AF-AMP activity inhibitor was found. AF-GGTP had a longer half-life than AF-AMP.

CONCLUSION: Due to the swallowing anomaly observed in EA, GGTP and AMP values physiologically observed at $18 \mathrm{wk}$ will decrease on the basis of the half-lives of markers, with a flat slope for GGTP and a sharp slope for AMP, therefore explaining the differences observed in the AF-EA pattern.

드ring ophageal atresia (EA) is a congenital malformation occurring in 1 of 3,500 live-births, characterized by disruption of the continuity of the esophagus associated or not with a tracheoesophageal fistula (1-3). Ladd's classification defines five subtypes (type I to type $\mathrm{V}$ ) on the basis of the location of the atresia and the type of fistula, type III (EA with distal fistula) accounting for $80 \%$ of cases (4). The pathophysiological mechanism leading to EA is not yet known and most probably involves genetic and environmental factors (5-7). The outcome of affected neonates may be improved by prenatal diagnosis allowing optimization of both prenatal and postnatal care. In EA, impaired fetal swallowing in conjunction with obstruction of the gastrointestinal tract contributes to the development of polyhydramnios and nonvisualizable fetal stomach (8). Therefore, the suspicion of EA is usually based on these findings at ultrasound with, however, a high false-positive rate and a poor detection rate of $\sim 50 \%(3,8-11)$. In a previous study based on a series of 44 cases of EA, we observed that amniotic fluid (AF) biochemistry had potential additional value in prenatal diagnosis, with a significant difference for total protein, $\alpha$-fetoprotein, and $\gamma$-glutamyl transpeptidase (GGTP) between the EA group (whatever the anatomical type) and the control group (12). These markers are part of AF biochemical patterns used for prenatal diagnosis of different gastrointestinal diseases as previously described $(13,14)$. The AF digestive enzymes (AF-DEs) we assayed, GGTP, L-leucine-aminopeptidase (AMP), and intestinal alkaline phosphatase (iALP), are secreted by microvilli. Previous studies of fetal tissues had specified the location of each enzyme: biliary epithelia for GGTP, proximal small bowel for AMP, and distal small bowel for iALP (14). AF-DE patterns are related to the physiological maturation of the fetal digestive tract. At 11-12 wk, the opening of pharyngeal membrane associated with AF swallowing leads to accumulation of the three studied DEs into the digestive tract, and then are secondary released in the AF when the anal membrane opens. Between 16 and $18 \mathrm{wk}$, due to a balance between swallowing and anal leakage, AF-DEs reach a plateau. After $18 \mathrm{wk}$, the maturation of the anal sphincter impairs this flow. Normal fetuses continue to swallow this AF charged with DEs, which are stocked in meconium up to birth, therefore explaining the decrease of these enzymes in the AF until delivery. AF-DE activities can be used in the prenatal diagnosis of fetal digestive malformations $(13,14)$. Briefly, when the fetus presents an upper tract digestive stenosis/atresia, induced vomiting floods the AF with proximal tract DEs (GGTP and AMP, but not iALP). Conversely, when the fetus presents a lowlevel obstruction, none of these enzymes are present in the

'Department of Pediatric Surgery, Robert Debré Hospital, AP-HP, University of Paris VII, Paris, France; ${ }^{2}$ Department of Biochemistry, Robert Debré Hospital, AP-HP, University of Versailles-Saint Quentin, Paris, France; ${ }^{3}$ Department of Developmental Biology, Robert Debré Hospital, AP-HP, University of Paris VII, Paris, France; ${ }^{4}$ Department of Obstetrics and Gynecology, Necker-Enfants Malades Hospital, AP-HP, University of Paris V, Paris, France; ${ }^{5}$ Department of Pediatric Surgery, Necker-Enfants Malades Hospital, AP-HP, University of Paris V, Paris, France; ${ }^{6}$ Department of Obstetrics and Gynecology, Robert Debré Hospital, AP-HP, University of Paris VII, Paris, France. Correspondence: Françoise Muller (francoise.muller@rdb.aphp.fr) 
AF. According to these data, the specific AF-DE dissociated biochemical pattern we observed in EA-high levels of GGTP and normal AMP-instead of both elevated as observed in the classic pattern of upper digestive tract obstruction-is not comprehensible.

The aim of this study was to find a pathophysiological explanation for this dissociated biochemical pattern observed in EA. Four objectives were sought as follows: (i) comparing AF markers between EA and other digestive tract atresias, (ii) determining local GGTP synthesis in the upper cul-de-sac of the esophagus, (iii) determining the presence of a specific AF-AMP activity inhibitor, and (iv) comparing AF-AMP and AF-GGTP half-lives.

\section{RESULTS}

\section{Comparison of AF Biochemical Patterns Between EA-Affected Fetuses and Two Anatomical Forms of Duodenal Atresia}

The results are shown in Table 1. Patients with EA presented with a pattern characterized by high GGTP values (median: 3.80 multiple of median (MoM) and normal AMP values (median: $1 \mathrm{MoM}$ ). Patients with DA-AOS (duodenal atresia located above the Oddi sphincter) presented with an amniotic biochemical pattern similar to EA, with high GGTP values (median: $5.26 \mathrm{MoM}$ ) and normal AMP value (median: 1 MoM). Patients with DA-BOS (duodenal atresia located above the Oddi sphincter) presented with a "bilious vomiting pattern" with high levels of both GGTP (median: $35 \mathrm{MoM}$ ) and AMP (median: $6 \mathrm{MoM})$, significantly different $(P<0.001)$ from the EA pattern.

In summary, the AF-DE pattern of EA is similar to the pattern observed in DA-AOS, but is different from the DA-BOS pattern.

\section{Local GGTP Synthesis in an Upper Cul-de-Sac of the Esophagus}

GGTP immunohistochemical staining of tissue from the upper cul-de-sac of the esophagus of the two EA-affected fetuses and the four neonates was similar to that of control fetal esophagus (Figure 1a,b) and was negative when compared with the staining observed in positive control tissue (Figure 1c). This demonstrated the absence of local synthesis of GGTP in the esophageal epithelium in EA.

\section{Specific AMP Inhibitor in the EA-AF}

The results are shown in Figure 2. A control AF sample was overcharged with an EA-AF sample, and sequential dilutions of the mixture were performed. Theoretical AMP activities are similar to the observed values (correlation coefficient $=0.99$; $P<0.01$ ), demonstrating the absence of AMP activity inhibitor in EA-AF. The discordance between AMP and GGTP activities is not due to a specific AMP inhibitor.

Table 1. Medians and ranges of AF biochemical markers in EA, duodenal atresia, and control cases

\begin{tabular}{|c|c|c|c|c|c|}
\hline & \multicolumn{3}{|c|}{ Pathological groups } & \multicolumn{2}{|c|}{ Control groups } \\
\hline & $\begin{array}{l}\text { Isolated EA } \\
\quad(n=31)\end{array}$ & $\begin{array}{l}\text { Duodenal atresia above } \\
\text { Oddi sphincter }(n=7)\end{array}$ & $\begin{array}{l}\text { Duodenal atresia below } \\
\text { Oddi sphincter }(n=14)\end{array}$ & $\begin{array}{l}\text { Polyhydramnios } \\
\qquad(n=100)\end{array}$ & $\begin{array}{c}\text { Nonpolyhydramnios } \\
(n=100)\end{array}$ \\
\hline \multirow[t]{2}{*}{ GGTP (MoM) } & $3.80^{*}$ & $5.26^{*}$ & $35.00^{*}$ & 0.95 & 1.00 \\
\hline & $(1.60-7.89)$ & $(1.60-18.73)$ & $(8.00-278.00)$ & $(0.29-3.05)$ & $(0.28-2.06)$ \\
\hline \multirow[t]{2}{*}{ AMP (MoM) } & 1.00 & 1.00 & $6.00^{*}$ & 0.80 & 1.00 \\
\hline & $(0.22-2.40)$ & $(0.44-2.00)$ & $(3.00-22.00)$ & $(0.20-2.63)$ & $(0.40-2.40)$ \\
\hline \multirow[t]{2}{*}{ Total protein (g/l) } & $6.40^{*}$ & $6.60^{*}$ & $7.40^{*}$ & 3.30 & 3.33 \\
\hline & $(3.60-9.30)$ & $(3.50-12.20)$ & $(4.80-13.70)$ & $(1.20-6.50)$ & $(1.20-9.50)$ \\
\hline
\end{tabular}

EA, esophageal atresia; MoM, multiple of median.

${ }^{*} P<0.001$ for comparison with the two control groups.

a

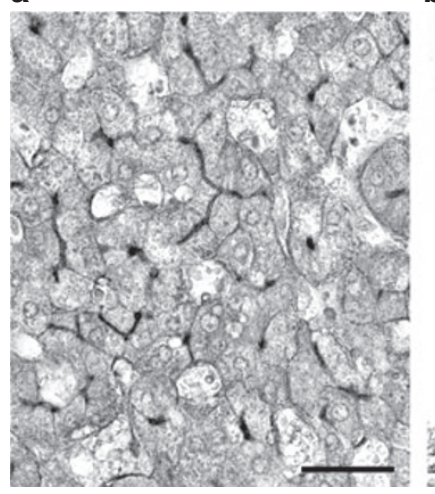

b

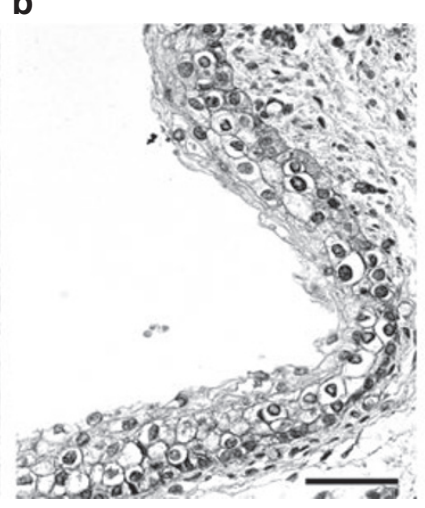

c

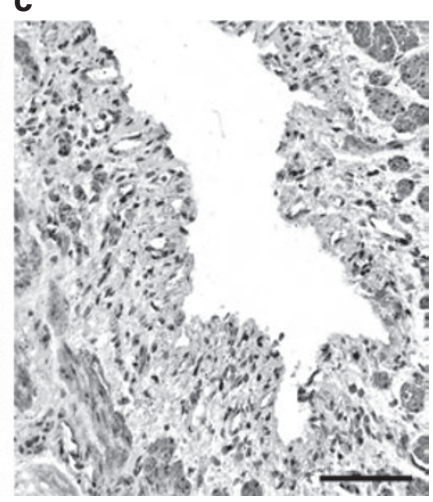

Figure 1. $\gamma$-Glutamyl transpeptidase (GGTP) immunostaining (magnification bar $=200 \mu \mathrm{m}$ ). (a) Positive control liver tissue for GGTP immunostaining. In the fetal liver, GGTP was localized in the bile canaliculi of the hepatocytes. The luminal surface of the biliary epithelium was also positive. (b) Negative control of normal fetal esophagus for GGTP immunostaining. (c) Negative GGTP immunostaining in the upper cul-de-sac of esophageal atresia tissue. 


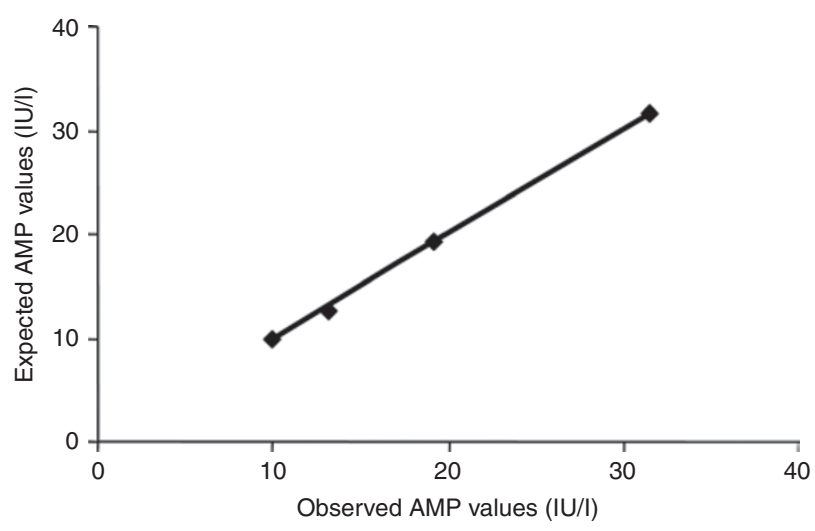

Figure 2. Regression curve for theoretical and observed aminopeptidase (AMP) activities. A control amniotic fluid (AF) sample was overcharged with an AF sample corresponding to an esophageal atresia-affected fetus. Sequential dilutions of the mixture were performed. Each point is the mean of four measurements (coefficient of variation $<3 \%$ ).

\section{Study of GGTP and AMP Half-Lives in AF}

The results are shown in Figure 3. The slopes of the two curves indicated a $40 \%$ decrease for AMP vs. a $12 \%$ decrease for GGTP. This, therefore, corresponded to a longer half-life of AF-GGTP. Figure 4 presents AF-GGTP and AMP kinetics as a function of gestational age in controls and in EA cases. The difference between the half-lives of GGTP and AMP explains the observed differences between GGTP and AMP in EA.

\section{DISCUSSION}

In a previous study (12), we demonstrated that the association of ultrasound scan screening and AF biochemistry showed 98\% sensitivity and $100 \%$ specificity in EA diagnosis. However, no comprehensible and plausible pathophysiological explanation was provided for the specific biochemical AF-EA pattern we observed associating high GGTP and normal AMP activities. In this study, on the basis of a defect in AF swallowing in EA fetuses, we demonstrated that the difference between the halflives of GGTP and AMP could explain the specific biochemical pattern in EA-affected fetuses. GGTP and AMP are not swallowed by the EA-affected fetus because of esophageal disruption and therefore stay in the $\mathrm{AF}$ at the level observed at $18 \mathrm{wk}$. The only decrease is due to the half-lives of the enzymes: a flat decrease for GGTP (longer half-life) and a sharper decrease for AMP (shorter half-life).

Because persistent swallowing disorders are observed after birth in EA-affected neonates and infants $(3,15)$ and because EA-affected fetuses cannot swallow AF, we first compared EA with the fetal swallowing anomalies observed in another upper tract intestinal obstruction (duodenal atresia, above or below the Oddi sphincter) in order to find a plausible pathophysiological explanation for the EA-dissociated biochemical pattern. We confirmed the parallel increase of both GGTP and AMP activity levels in DA-BOS (13) and observed that DA-AOS showed an AF pattern different from DA-BOS but identical to that of EA. The difference in GGTP levels between DA-BOS and DA-AOS is anatomically comprehensible because GGTP synthesis occurs mostly in the biliary epithelium. However, the

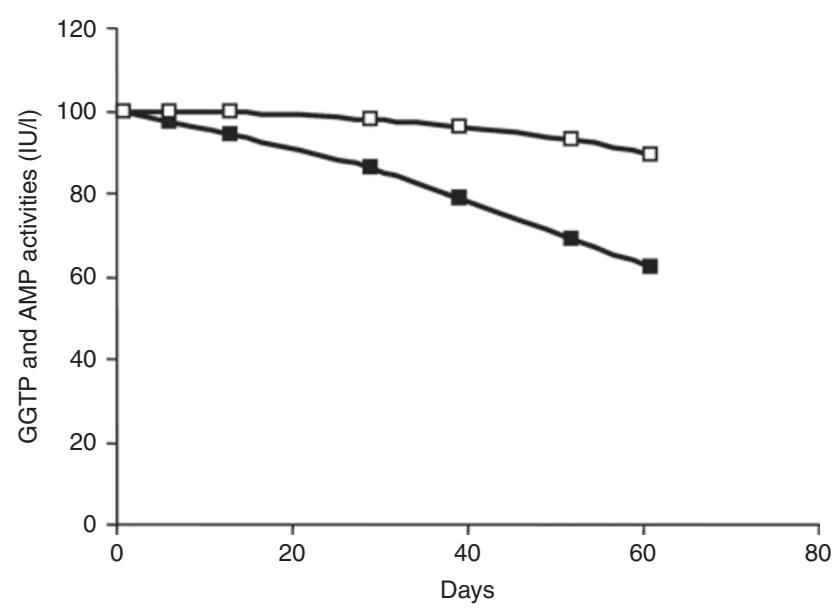

Figure 3. Amniotic fluid (AF) $Y$-glutamyl transpeptidase (GGTP, white squares) and aminopeptidase (AMP, black squares) activities as a function of time, ex vivo. A pool of four AFs was kept at $37^{\circ} \mathrm{C}$ for up to $60 \mathrm{~d}$. Aliquots were tested at 7- to 10-day intervals.

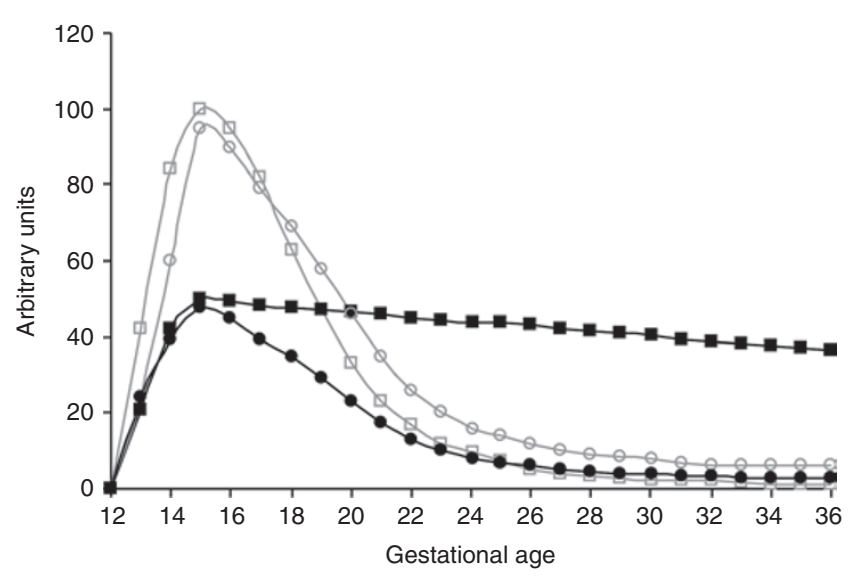

Figure 4. Theoretical model of amniotic fluid (AF) $\gamma$-glutamyl transpeptidase (black and gray squares) and aminopeptidase (black and gray circles) values expressed in arbitrary units as a function of gestational age (weeks of gestation). Gray lines represent values observed in controls and bold lines represent values observed in esophageal atresia (EA) cases. In controls and in EA cases, anal membrane opens at 12 wk. Digestive enzymes are massively released in the AF but at a lower level in EA because of the swallowing defect. Due to anal sphincter maturation beginning around 16-20 wk, digestive enzymes are swallowed and stocked in the meconium until delivery. However, in EA cases, due to the anatomical lesions and swallowing defect, digestive enzymes stay in the AF with a slope of decrease only depending on their own half-life.

high GGTP levels, even if much lower than in a bilious vomiting pattern, were not be comprehensible. We also excluded the presence of an AMP inhibitor in the EA-AF. Finally, after exclusion of metaplastic synthesis of GGTP in the upper culde-sac of the esophagus, the only valid hypothesis was the difference in half-life between GGTP and AMP. The two enzymes present in the AF after physiological opening of the anal membrane (12-14 SA) are not swallowed by the fetus because of the esophageal disruption, and stay in the $\mathrm{AF}$ at the maximum levels observed at the 16-18wk. Because AMP has a shorter half-life than GGTP, its degradation occurs faster: $1 \mathrm{MoM}$ for AMP at 25 wk as compared with 3 MoM for GGTP. However, 
a

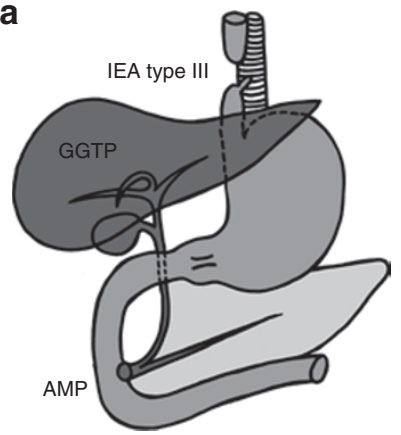

b

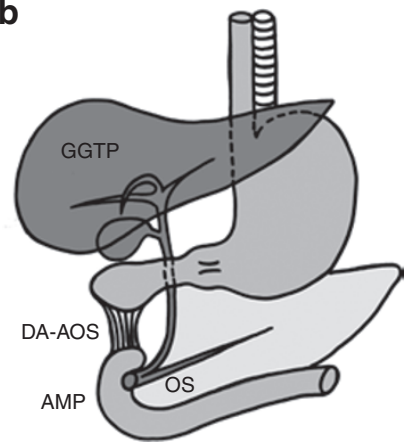

C

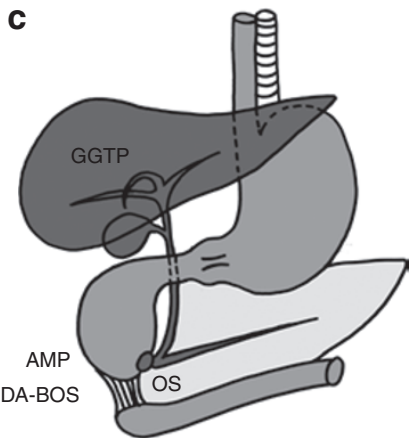

Figure 5. Anatomical description of esophageal atresia type III and of the two types of duodenal atresia. (a) Anatomical description of esophageal atresia (EA) with tracheoesophageal fistula (type III). (b) Anatomical description of duodenal atresia located above the Oddi sphincter. (c) Anatomical description of duodenal atresia located below the Oddi sphincter. AMP, aminopeptidase; AMP M, aminopeptidase M; DA-AOS, duodenal atresia located above the Oddi sphincter; DA-BOS, duodenal atresia located below the Oddi sphincter; GGTP, $\gamma$-glutamyl transpeptidase; IEA, isolated EA; OS, Oddi sphincter.

it is impossible to firmly exclude the presence of a labile inhibitor not measurable in vitro due to its fast degradation. Then we tried to explain the high levels of GGTP in AF of EA-affected fetuses.

The different half-lives of GGTP and AMP can probably be explained by their molecular structures. GGTP is an extracellular enzyme anchored to the membrane of cells, playing a central role in glutathione metabolism with no variation in glycosylation in humans. GGTP is found in organisms from bacteria to mammals and plants. Literature data concerning GGTP biochemical properties showed evidence of high thermostability, independence of $\mathrm{pH}$ variations, and stability in high-salt conditions (16-21). AMPs comprise a large family of biologically important zinc enzymes playing different roles in the regulation of blood pressure, antigen presentation, memory, cell cycle, pregnancy, and angiogenesis. Numerous polymorphisms are present within the human species, and mutations can result in hypertension or preeclampsia $(22,23)$. Literature data are scarce concerning the biochemical properties of AMP, notably its biochemical stability is not reported and reports mostly focused on its multiple polymorphisms and their consequences for the phenotype. We may imagine that its great variability and the specificity of its physiological role in the human species point to its fragility and short half-life in $\mathrm{AF}$.

In conclusion, the pathophysiological explanation we give for the specific AF biochemical pattern we observed in EA provides a solid understanding of the good detection rate and specificity we observed. This may lead on to comprehension of other swallowing anomalies during the prenatal period. In addition, when the AF- DE profile is suggestive of EA, this is necessarily related to a swallowing defect present as early as $16 \mathrm{wk}$ of gestation. This very early swallowing defect would explain the severe swallowing difficulties observed after birth with their nutritional consequences. Positive biochemical diagnosis of EA would therefore signify severe consequences. Conversely, when prenatal diagnosis is not performed because of the absence of ultrasound signs, the swallowing anomalies probably do not exist prenatally and likely correspond to less severe swallowing anomalies after birth. Another study is needed to demonstrate the relation between absence of prenatal signs and postnatal swallowing capacity.

\section{METHODS}

\section{Patients and AF Samples}

We retrospectively compared the AF biochemical pattern of fetal digestive tract anomalies and controls. Samples of AF were sent during the period 2003-2009 from different institutions to our laboratory for biochemistry assay. Fetal karyotyping was performed in all cases and was normal. In accordance with French law, consent for amniocentesis and for laboratory testing was obtained from each patient. Anatomical lesions were diagnosed postnatally at anatomical examination or at surgical treatment. Four groups were compared. Group 1 consisted of a series of 31 cases of isolated EA, prenatally suspected at routine second-trimester ultrasound screening based on polyhydramnios and/or nonvisualization of the stomach bubble. Amniocentesis was performed in all cases after 23 wk of gestation. Anatomical lesions were of type I $(n=12)$, type II $(n=1)$, type III $(n=15)$, type IV $(n=2)$, or type V $(n=1)$. Group 2 consisted of a series of 21 cases of duodenal atresia prenatally suspected at ultrasound scan based on the presence of a double bubble image and polyhydramnios. Anatomical lesions were located above the Oddi sphincter (DA-AOS) $(n=7)$ or below (DA-BOS) $(n=14)$ as displayed in Figure 5a-c. Group 3 corresponded to an idiopathic polyhydramnios group $(n=100)$, and Group 4 to a nonpolyhydramnios control group $(n=100)$ sampled for fetal karyotyping because of maternal age ( $38 \mathrm{y}$ and over) or because of Down syndrome risk $>1$ in 250 at maternal serum marker screening. Twin pregnancies, fetal aneuploidy, oligohydramnios, fetal morphological abnormalities, and cases with a clear etiology of polyhydramnios were excluded from controls.

\section{AF Biochemistry}

Four biochemical markers were assayed: total protein (urinary/cereberospinal fluid protein; Beckman Coulter, Fullerton, CA) expressed in $\mathrm{g} / \mathrm{l}$ and DE activities (AU400; Beckman Coulter), including GGTP, AMP, and iALP. Normal values of these markers had previously been established as a function of gestational age (14). Results are expressed in MoM, corresponding to the ratio between the observed raw value and the median raw value defined for the same gestational age. For the study of GGTP and AMP half-lives in AF, we used four pools of control AF samples at different GGTP levels (200, 500, 700, and 1000 $\mathrm{IU} / \mathrm{l})$ and AMP levels $(20,60,80$, and $100 \mathrm{IU} / \mathrm{l})$ incubated at $37^{\circ} \mathrm{C}$. An aliquot was tested at 7- to 10-day intervals for AMP and GGTP activities up to $61 \mathrm{~d}$. Finally, based on previous data (14) and on the GGTP and AMP half-lives we observed herein, we have constructed a theoretical model explaining AF-GGTP and AMP kinetics as a function of gestational age in controls and in EA cases. 


\section{Tissue GGTP Activity Immunohistochemical Staining}

GGTP synthesis in the upper cul-de-sac of the esophagus was studied in two fetuses sampled during pathological examination (vertebral defects, anal defects, cardiac defects, tracheoesophageal fistula, renal defects, limb defects, and VACTERL association) and in four specimens sampled during surgical repair at day 1 after birth. They were compared with normal fetal esophageal and tracheal tissues (negative control) and with fetal testis, thyroid, and liver tissues (positive control) (24). Formalin-fixed paraffin-embedded tissues were obtained from archival autopsy. Sections of $5 \mu \mathrm{m}$ were immunostained using a primary monoclonal anti-GGTP antibody (1:2,000 dilution; Applied Cell Biotechnologies, Yokohama, Japan). The LSAB2 streptavidinbiotin detection system kit (Dako, Trappes, France) was used to reveal immunolabeling as previously described (25). The slides were counterstained with Mayer's hemalum solution and mounted in PERTEX medium (Histolab Products, Gothenburg, Sweden).

\section{Statistical Analysis}

The nonparametric Mann-Whitney test was used for group comparisons. $P<0.05$ was considered as significant. A correlation coefficient (Pearson) was used for correlation between markers, and $P<0.05$ was considered as significant. The study was exempt from institutional review board approval because at the time of prenatal diagnosis AF sampling was part of the routine diagnostic work-up.

\section{STATEMENT OF FINANCIAL SUPPORT}

No financial assistance was received to support this study.

Disclosure: No financial ties disclosures and no conflicts of interest.

\section{REFERENCES}

1. Depaepe A, Dolk H, Lechat MF. The epidemiology of tracheo-oesophageal fistula and oesophageal atresia in Europe. EUROCAT Working Group. Arch Dis Child 1993;68:743-8.

2. Torfs CP, Curry CJ, Bateson TF. Population-based study of tracheoesophageal fistula and esophageal atresia. Teratology 1995;52:220-32.

3. Sparey C, Jawaheer G, Barrett AM, Robson SC. Esophageal atresia in the Northern Region Congenital Anomaly Survey, 1985-1997: prenatal diagnosis and outcome. Am J Obstet Gynecol 2000;182:427-31.

4. Spitz L. Oesophageal atresia. Orphanet J Rare Dis 2007;11:2-24.

5. Geneviève D, de Pontual L, Amiel J, Sarnacki S, Lyonnet S. An overview of isolated and syndromic oesophageal atresia. Clin Genet 2007;71:392-9.

6. Oddsberg J, Lu Y, Lagergren J. Aspects of esophageal atresia in a population-based setting: incidence, mortality, and cancer risk. Pediatr Surg Int 2012;28:249-57.

7. Felix JF, de Jong EM, Torfs CP, de Klein A, Rottier RJ, Tibboel D. Genetic and environmental factors in the etiology of esophageal atresia and/or tracheoesophageal fistula: an overview of the current concepts. Birth Defects Res Part A Clin Mol Teratol 2009;85:747-54.

8. Houben $\mathrm{CH}$, Curry JI. Current status of prenatal diagnosis, operative management and outcome of esophageal atresia/tracheo-esophageal fistula. Prenat Diagn 2008;28:667-75.

9. de Jong EM, Felix JF, de Klein A, Tibboel D. Etiology of esophageal atresia and tracheoesophageal fistula: "mind the gap". Curr Gastroenterol Rep 2010;12:215-22.
10. Langer JC, Hussain H, Khan A, et al. Prenatal diagnosis of esophageal atresia using sonography and magnetic resonance imaging. J Pediatr Surg 2001;36:804-7.

11. Stringer MD, McKenna KM, Goldstein RB, Filly RA, Adzick NS, Harrison MR. Prenatal diagnosis of esophageal atresia. J Pediatr Surg 1995;30:125863.

12. Czerkiewicz I, Dreux S, Beckmezian A, et al. Biochemical amniotic fluid pattern for prenatal diagnosis of esophageal atresia. Pediatr Res 2011;70:199-202.

13. Muller F, Dommergues M, Ville $\mathrm{Y}$, et al. Amniotic fluid digestive enzymes: diagnostic value in fetal gastrointestinal obstructions. Prenat Diagn 1994;14:973-9.

14. Muller F, Oury JF, Dumez Y, Boué J, Boué A. Microvillar enzyme assays in amniotic fluid and fetal tissues at different stages of development. Prenat Diagn 1988;8:189-98.

15. Hanigan MH, Frierson HF Jr. Immunohistochemical detection of gammaglutamyl transpeptidase in normal human tissue. J Histochem Cytochem 1996;44:1101-8.

16. Guimiot F, Marcorelles P, Aboura A, et al. Giant diencephalic harmartoma and related anomalies: a newly recognized entity distinct from the Pallister-Hall syndrome. Am J Med Genet A 2009;149A: $1108-15$

17. Sistonen SJ, Pakarinen MP, Rintala RJ. Long-term results of esophageal atresia: Helsinki experience and review of literature. Pediatr Surg Int 2011;27:1141-9.

18. West MB, Segu ZM, Feasley CL, et al. Analysis of site-specific glycosylation of renal and hepatic $\gamma$-glutamyl transpeptidase from normal human tissue. J Biol Chem 2010;285:29511-24.

19. Castellano I, Merlino A, Rossi M, La Cara F. Biochemical and structural properties of gamma-glutamyl transpeptidase from Geobacillus thermodenitrificans: an enzyme specialized in hydrolase activity. Biochimie 2010;92:464-74.

20. Yang JC, Liang WC, Chen YY, et al. Biophysical characterization of Bacillus licheniformis and Escherichia coli $\gamma$-glutamyltranspeptidases: a comparative analysis. Int J Biol Macromol 2011;48:414-22.

21. Wada K, Irie M, Suzuki H, Fukuyama K. Crystal structure of the halotolerant gamma-glutamyltranspeptidase from Bacillus subtilis in complex with glutamate reveals a unique architecture of the solvent-exposed catalytic pocket. FEBS J 2010;277:1000-9.

22. Chang HP, Liang WC, Lyu RC, et al. Effects of C-terminal truncation on autocatalytic processing of Bacillus licheniformis gamma-glutamyl transpeptidase. Biochemistry Mosc 2010;75:919-29.

23. Heisterkamp N, Groffen J, Warburton D, Sneddon TP. The human gammaglutamyltransferase gene family. Hum Genet 2008;123:321-32.

24. Tsujimoto M, Goto Y, Maruyama M, Hattori A. Biochemical and enzymatic properties of the M1 family of aminopeptidases involved in the regulation of blood pressure. Heart Fail Rev 2008;13: 285-91.

25. Tholander F, Muroya A, Roques BP, Fournié-Zaluski MC, Thunnissen MM, Haeggström JZ. Structure-based dissection of the active site chemistry of leukotriene A4 hydrolase: implications for M1 aminopeptidases and inhibitor design. Chem Biol 2008;15:920-9. 